\title{
Integrating protein localization with automated signaling pathway reconstruction
}

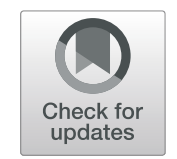

\author{
Ibrahim Youssef ${ }^{1,2}$, Jeffrey Law ${ }^{3}$ and Anna Ritz ${ }^{2 *}$ \\ From IEEE International Conference on Bioinformatics and Biomedicine 2018 \\ Madrid, Spain. 3-6 December 2018
}

\begin{abstract}
Background: Understanding cellular responses via signal transduction is a core focus in systems biology. Tools to automatically reconstruct signaling pathways from protein-protein interactions (PPIs) can help biologists generate testable hypotheses about signaling. However, automatic reconstruction of signaling pathways suffers from many interactions with the same confidence score leading to many equally good candidates. Further, some reconstructions are biologically misleading due to ignoring protein localization information.

Results: We propose $\mathrm{LOCPL}$, a method to improve the automatic reconstruction of signaling pathways from PPIs by incorporating information about protein localization in the reconstructions. The method relies on a dynamic program to ensure that the proteins in a reconstruction are localized in cellular compartments that are consistent with signal transduction from the membrane to the nucleus. LOCPL and existing reconstruction algorithms are applied to two PPI networks and assessed using both global and local definitions of accuracy. LocPL produces more accurate and biologically meaningful reconstructions on a versatile set of signaling pathways.

Conclusion: LOCPL is a powerful tool to automatically reconstruct signaling pathways from PPIs that leverages cellular localization information about proteins. The underlying dynamic program and signaling model are flexible enough to study cellular signaling under different settings of signaling flow across the cellular compartments.
\end{abstract}

Keywords: Signaling pathways, Biological networks, Protein-protein interaction, Protein localization

\section{Background}

A fundamental goal of molecular systems biology is to understand how individual proteins and their interactions may contribute to a larger cellular response. Repositories for experimentally derived or manually curated human protein-protein interaction (PPI) information [1-7] have been critical for achieving that goal. These databases conceptualize the interaction information as a graph, or an interactome, where edges connect proteins that are known to interact. Such interactomes are useful for studying the topology of signaling pathways by forming static networks and focusing on the interconnections between proteins and how signals flow between them. In particular, interaction data have enabled the development of methods that

*Correspondence: aritz@reed.edu

${ }^{2}$ Biology Department, Reed College, Portland OR 97202, USA

Full list of author information is available at the end of the article aim to link extracellular signals to downstream cellular responses.

Most methods that link signals with responses were initially applied to yeast studies [8-10]. A handful of the initial methods were applied to human signaling, including the apoptosis pathway [11] and the immune response network [12]. Approaches for identifying relevant static sub-networks have drawn on different graph theoretic methods, including shortest paths [13, 14], Steiner trees and related formulations $[15,16]$, network flow $[9,17]$ and random walk approaches [18-20].

As the wealth of PPI information has grown, these methods have been increasingly adopted to study human signaling. PathLinker is a recent pathway reconstruction approach that returns ranked paths for a specific human signaling pathway of interest [13]. Given a weighted interactome, a set of known receptors, and a set of known transcriptional regulators (TRs), PathLinker returns the 
$k$-shortest paths from any receptor to any transcriptional regulator, and the collection of these paths constitute a pathway reconstruction. PathLinker reconstructions have been shown to outperform other pathway reconstruction methods on human networks [13]. PathLinker predicted that CFTR, a chloride ion channel transporter, was involved in Wnt signaling; RNAi and Co-immunoprecipitation experiments confirmed CFTR's involvement in Wnt signaling in HEK293 cells [13].

Pathway Reconstruction Challenges. Despite PathLinker's success, the problem of identifying accurate pathway reconstructions remains challenging. PathLinker paths are prioritized by their reconstruction scores that are the product of a path edge weights. These paths combined form a pathway reconstruction. We assessed PathLinker reconstructions for four well-studied and diverse signaling pathways: the Wnt pathway is critical for the development of tissues cell fate specification [21]; the Interleukin-2 (IL2) pathway plays a major role in controlling the immune system and regulating homeostasis [22]; the $\alpha 6 \beta 4$ Integrin pathway regulates cell adhesion to the extracellular matrix [23]; and the Epidermal Growth Factor Receptor (EGFR1) pathway regulates cell proliferation, survival, and migration [24]. Careful analysis of the ranked paths across these pathways revealed two main challenges in pathway reconstruction.

First, we found that many PathLinker paths have identical reconstruction scores. For example, about $52 \%$ of the paths in the Wnt reconstruction had the same score. This feature was not unique to Wnt; $64 \%, 82.6 \%$, and $48.2 \%$ of the paths were tied in the IL2, $\alpha 6 \beta 4$ Integrin, and EGFR1 pathways, respectively. Strikingly, even the topranked paths in the reconstructions were often tied (top 38 paths in Wnt, top 87 paths in IL2, top 57 paths in $\alpha 6 \beta 4$ Integrin, and top 330 paths in EGFR1). We found that the tied paths were a result of many interactions with identical weights in the underlying interactome (Fig. 1). For example, in the PathLinker interactome (PLNet 1 ), nearly $68 \%$ of the interactions have only two distinct weight values. In the interactome used in this work ( LLNet $\left._{2}\right)$, around $71 \%$ of the interactions have just three different weight values. The coarse interaction weighting is also apparent in the HIPPIE network [2], where 55\% of the interactions share the same edge weight (Fig. 1).

Second, we noted that paths in the reconstructions contained a mix of pathway-specific signaling interactions relevant to the pathway under study (positive interactions) and non-pathway interactions (we will call them negative interactions, though they may very well be signaling interactions relevant to other pathways or pathway-specific interactions that have not been annotated yet). Paths are rarely comprised solely of positive interactions: in all four pathway reconstructions, over $95 \%$ of the paths that include at least one positive interaction also contain a negative interaction. PathLinker does not consider protein localization in the pathway reconstructions, so interactions within the same path may be unrealistic in terms of compartment co-localization. Given the first challenge

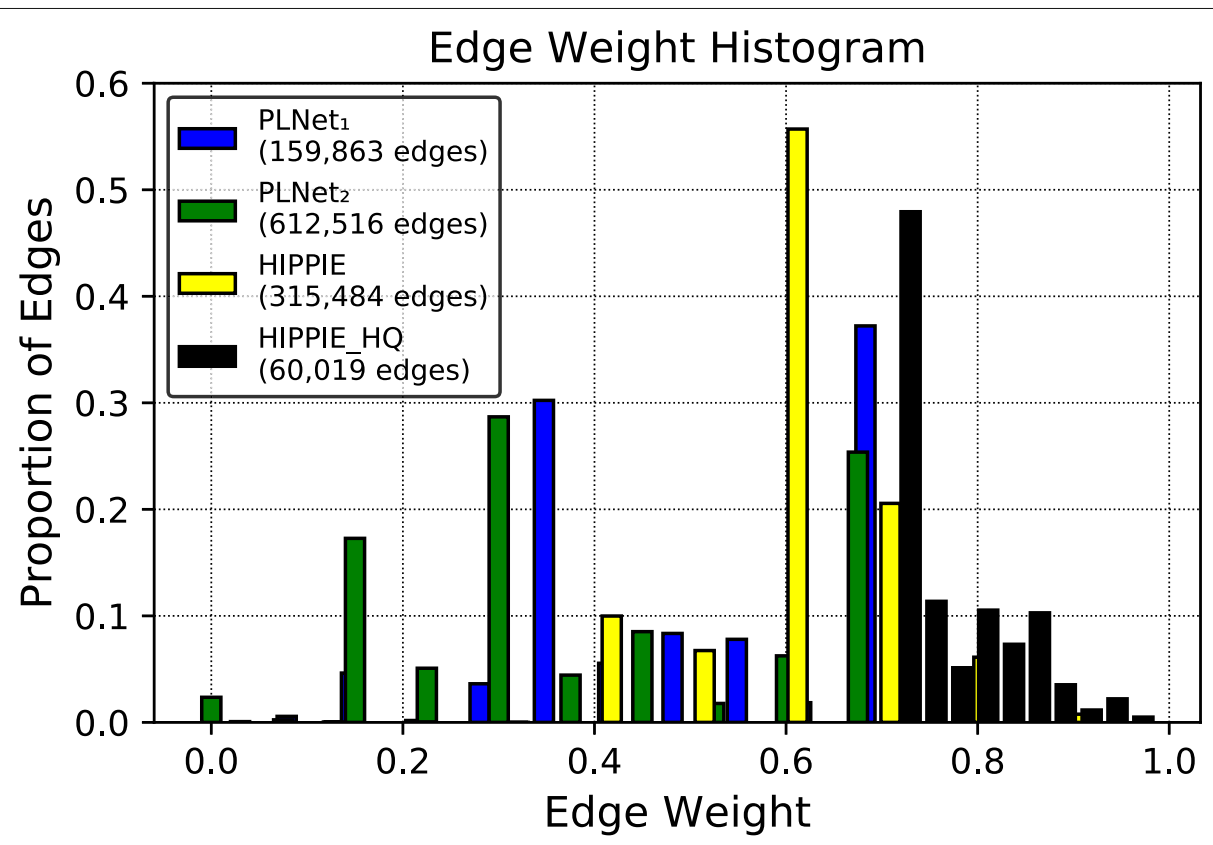

Fig. 1 Proportion of edges with identical edge weights in the PathLinker and HIPPIE interactomes. PLNet $t_{1}$ is the PathLinker interactome [13], while PLNet 2 is the interactome used in this work. The HIPPIE High Quality (HIPPIE_HQ) interactome includes all HIPPIE edges with a weight $\geq 0.73$ [2]. The histogram number of bins is 10 with a size of 0.02 for each 
of coarse interaction weights, additional evidence about protein localization could be useful for breaking tied path scores.

To overcome the challenges described above, we sought to incorporate an independent data type into the pathway reconstruction problem. While many methods have integrated gene expression data in pathway reconstructions $[9,15,20]$, we wish to improve "canonical" pathways that are independent of a specific context (e.g. a condition or disease). Instead, we make use of information about a protein's localization within the cell to constrain the paths in a reconstruction.

Contributions. We propose LocPL, an extended version of PathLinker that reconstructs pathways by incorporating information about cellular localization in two ways. First, LocPL uses localization information to discard likely false positive interactions from the interactome before running PathLinker, improving its specificity. Second, LocPL incorporates the localization information in a dynamic programming scheme to identify spatiallycoherent paths and re-prioritize tied paths (Fig. 2a). We show that paths with larger proportions of signaling interactions will be promoted higher in the $k$-shortest paths list, and those of smaller proportions will be demoted. We compare the LocPL pathway reconstructions to those from PathLinker on two interactomes: a new interactome, $\mathrm{PLNet}_{2}$, which quadruples the number of interactions compared to the PathLinker interactome, and the HIPPIE interactome [2]. We also compare LocPL to a color-coding method $[25,26]$. In addition to performing a global performance assessment of paths, we present a local measure to assess path quality individually. Visual inspection of the top 100 paths in the Wnt, IL2, $\alpha 6 \beta 4$ Integrin, and EGFR1 pathway reconstructions reveal that the spatially-coherent approach changes the reconstruction topology, in some cases removing paths that lead to activation of other pathways. This work demonstrates that incorporating protein localization information into signaling pathway reconstruction improves predictions that are necessary for appropriate hypothesis generation.

\section{Methods}

We first introduce ComPPI, the protein localization database that $L o c P L$ uses to refine pathway reconstructions, and then we present an overview of LocPL. After describing the model used for signaling flow, we present a dynamic program for computing scores that reflect a path's consistency with the model of signaling. Then, we describe the color-coding method that LocPL is compared to. Finally, we detail the interactome and signaling pathway datasets and the means of assessing pathway reconstruction performance.

\section{Localized protein-protein interactions from ComPPI}

ComPPI is a database that predicts cellular compartments for human proteins and PPIs [27] (Version 2.1.1, September $10^{\text {th }}, 2018$ [28]). For each protein, ComPPI computes localization scores describing the likelihood of a protein to be found in one of the major six subcellular

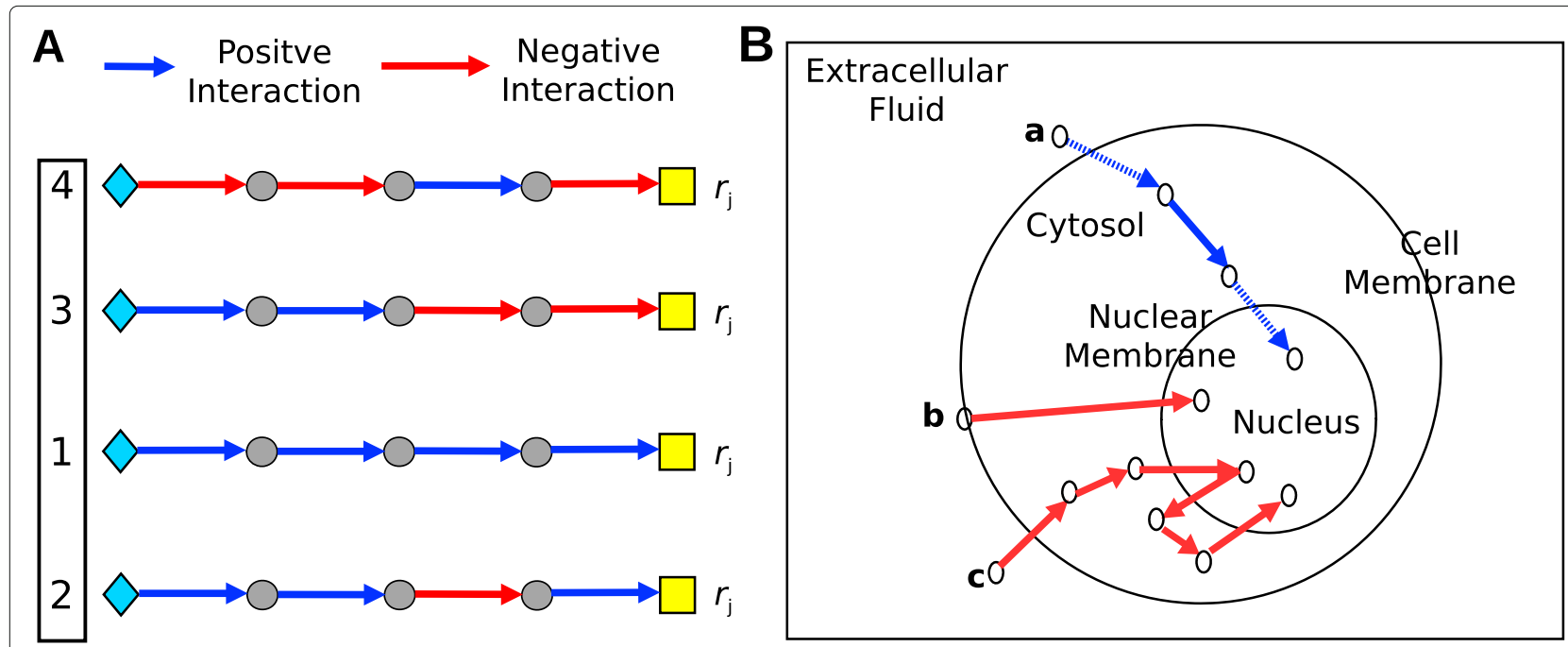

Fig. 2 a lllustration of four PathLinker paths from receptors (diamonds) to transcriptional regulators (yellow boxes) that all have the same reconstruction score $r_{j}$. Blue edges represent true positive interactions, and red edges represent false positives. The goal of breaking ties is to re-rank the tied paths so paths with more positives are ranked higher (black box). b Simplified model diagram for the signaling flow structure. Blue edges represent valid interactions. The blue solid edges are between pairs of proteins sharing one cellular compartment, and the blue dotted edges are proteins that traverse between two compartments. Paths that violate our signaling model assumptions are shown in red, where path (b) has a single interaction between a pair of proteins without a common cellular compartment, and signaling in path (c) does not reside in the nucleus once it reached the nuclear compartment 
compartments: (i) extracellular fluid, (ii) cell membrane, (iii) cytosol, (iv) nucleus, (v) secretory pathway (e.g. transport vesicles), and (vi) mitochondria. ComPPI uses three types of information to infer the localization scores: experimental verification, computational prediction, and unknown sources, resulting in high, medium, and low localization scores, respectively. The interaction score, computed by ComPPI from localization scores of the participating proteins, represents the probability that an interaction takes place inside the cell.

\section{LocPL: localized pathLinker}

Signaling pathway analysis methods typically take an interactome as input, represented as a graph $G=(V, E)$ where the nodes $V$ are proteins and the edges $E$ are PPIs. In the case of $L o c P L$, the graph is directed, each edge $(u, v) \in E$ has a weight $w_{u v} \in[0,1]$, and every interaction is predicted to occur within some cellular compartment according to ComPPI. LocPL uses the ComPPI database to restrict the interactions of the interactome by removing edges with an interaction score of zero - these interactions could take place from a biophysical perspective, but are less likely to occur within the cell due to the predicted protein localization. After this filtration step, all edges in the interactome have a non-zero probabilistic score aggregated across all cellular compartments. For subsequent steps of $L o c P L$, we use the ComPPI localization scores that reflect individual proteins in specific cellular compartments.

LocPL's core method is a $k$-shortest path algorithm previously described as PathLinker [13]. Given a directed, weighted interactome $G$, a set $R$ of receptors and a set $T$ of transcriptional regulators (TRs) for a pathway of interest, and a number of paths $k$, PathLinker outputs a ranked list of the $k$ shortest paths, $\mathcal{P}=\left\langle P_{1}, P_{2}, \ldots, P_{k}\right\rangle$, where a path $P_{i}=\left(v_{1}, v_{2}, \ldots, v_{m}\right)$ is comprised of $m$ nodes that begin at a receptor $\left(v_{1} \in R\right)$ and ends at a TR $\left(v_{m} \in T\right)$. Each path $P_{i}$ is ranked by the product of its edge weights (its reconstruction score $r_{i}$ ), and $r_{i} \geq r_{i+1}$ for every $i$. Note that the shortest path is the one whose edge weights product is the highest among all paths since PathLinker takes the negative log-transform of the edge weights at the reconstruction step.

After running PathLinker on the interactome, LocPL breaks ties in the candidate list of paths $\mathcal{P}$ by considering a model of signaling flow based on cellular compartments. For each path $P_{i}$, a dynamic program identifies the signaling score $s_{i}$ of the most likely series of compartments for each node that is consistent with the signaling flow model. After this step, each path $P_{i}$ will have two scores: a reconstruction score $r_{i}$ computed by PathLinker and a signaling score $s_{i}$ computed by the dynamic program. The signaling score is used to re-prioritize the tied reconstruction scores by partitioning the paths into ties (e.g. all paths with the same reconstruction score) and reordering the paths within each group in decreasing order of the signaling score (Fig. 2a).

\section{Signaling flow structure and assumptions}

In order to use protein localization information in pathway reconstructions, we first state some assumptions about the pathways we aim to reconstruct. First, we only consider intracellular signaling that begins with activation of a membrane-bound protein receptor and is transmitted to a DNA-binding transcription factor through PPIs within the cytosol. Hence, we focus on three cellular compartments: a combination of extracellular fluid and cell membrane (ExtMem), which represents where a receptor may be located, Cytosol, and Nucleus. Second, we assume a unidirectional signaling flow from ExtMem through Cytosol to Nucleus. Third, multiple interactions may occur within the same cellular compartment (e.g. multiple interactions may occur within Cytosol). Fourth, signaling flow advances through either interacting proteins that share the same cellular compartment, or a protein that can traverse different cellular compartments. These assumptions impose an ordering on the compartments that must be visited, which we will use in breaking tied paths. Figure $2 \mathrm{~b}$ illustrates these assumptions with three different paths as examples of valid and invalid paths/interactions. Path a is valid; however, path $\mathbf{b}$ is not valid because signaling goes directly from the cellular membrane to the nucleus and path $\mathbf{c}$ has one invalid interaction because signaling goes in a direction against the assumed signaling flow.

We acknowledge that the assumptions in this work may not hold for many pathways. For example, some pathways are initiated via nuclear receptors, and would be missed based on our assumption that signaling begins at receptors at the cell membrane. We also do not consider other compartments beyond ExtMem, Cytosol, and Nucleus in our model, while the mitochondria and secretory vesicles play an important role in some signaling pathways. These decisions can be taken by the user, which makes the proposed model of signaling flow customizable to a pathway under study. A priori information about the structure of signaling flow may further improve LocPL predictions.

\section{Dynamic program for path-based signaling scores}

Given a path $P=\left(v_{1}, v_{2}, \ldots, v_{m}\right)$ that connects $m$ proteins, our goal is to find a selection of compartments that maximize the path signaling score (by sum of log-transformed localization scores) while respecting the assumed signaling flow structure outlined above. For each protein $v \in V$, we use $\ell_{v}^{e x t}, \ell_{v}^{c y t}$, and $\ell_{v}^{n u c}$ to denote the ComPPI scores of ExtMem, Cytosol, and Nucleus respectively. We log-transform these scores to be localization costs, that is, $\ell_{v}^{c}=-\log \ell_{v}^{c}$ for each protein $v$ and each cellular compartment $c$ (either ExtMem, Cytosol, or 
Nucleus). Let $s\left(v_{j}, c\right)$ be the optimal score of the path up to node $v_{j} \in P$, where $v_{j}$ is in compartment $c$. The optimal signaling score of the path must end in the nucleus, which we denote by $s\left(v_{m}, n u c\right)$. Since our assumed signaling model requires that signaling advances through pairs of interacting proteins sharing a cellular compartment or through proteins that traverse multiple compartments, there are only three routes for the signaling information to advance from protein $v_{m-1}$ to end up in the nucleus for protein $v_{m}: 1$ ) protein $v_{m-1}$ and protein $v_{m}$ interact in the cytosol and then protein $v_{m}$ moves to the nucleus, 2) protein $v_{m-1}$ moves from the cytosol to the nucleus and then interacts with protein $v_{m}$ in the nucleus, or 3) protein $v_{m-1}$ and protein $v_{m}$ interact in the nucleus. Based on these constraints, the optimal path signaling score $s\left(v_{m}, n u c\right)$ can be computed as:

$$
\begin{aligned}
& s\left(v_{m}, n u c\right)=\min \left[s\left(v_{m-1}, c y t\right)+\ell_{v_{m}}^{c y t},\right. \\
& \left.s\left(v_{m-1}, c y t\right)+\ell_{v_{m-1}}^{n u c}, s\left(v_{m-1}, n u c\right)\right]+\ell_{v_{m}}^{n u c} .
\end{aligned}
$$

In general, at node $v_{j}, j=2,3, \ldots,(m-1)$, the set of equations for the scores are:

$$
\begin{aligned}
& s\left(v_{j}, \text { ext }\right)=s\left(v_{j-1}, \text { ext }\right)+\ell_{v_{j}}^{\text {ext }} \\
& s\left(v_{j}, c y t\right)=\min \left[s\left(v_{j-1}, \text { ext }\right)+\ell_{v_{j}}^{\text {ext }},\right. \\
& \left.s\left(v_{j-1}, \text { ext }\right)+\ell_{v_{j-1}}^{c y t}, s\left(v_{j-1}, c y t\right)\right]+\ell_{v_{j}}^{c y t} \\
& s\left(v_{j}, n u c\right)=\min \left[s\left(v_{j-1}, c y t\right)+\ell_{v_{j}}^{c y t},\right. \\
& \left.s\left(v_{j-1}, c y t\right)+\ell_{v_{j-1}}^{n u c}, s\left(v_{j-1}, n u c\right)\right]+\ell_{v_{j}}^{n u c} .
\end{aligned}
$$

Note that we can only reach a protein in ExtMem from another protein in ExtMem, we can reach a protein in Cytosol from another protein in either ExtMem or Cytosol, and we can reach a protein in Nucleus from another one in either Cytosol or Nucleus.

To ensure that the path starts with the cellular compartment ExtMem, the base case for these recurrence relations are:

$$
\begin{gathered}
s\left(v_{1}, \text { ext }\right)=\ell_{v_{1}}^{e x t} \\
s\left(v_{1}, c y t\right)=\infty \\
s\left(v_{1}, n u c\right)=\infty .
\end{gathered}
$$

The final score taken will be $s\left(v_{m}, n u c\right)$ since we require the path to terminate in the nucleus. These recurrence relations can be calculated using a dynamic program in linear time w.r.t. the path length for each tied path. An illustrative example of this dynamic program is provided in Additional file 1.

\section{The color-coding-based method}

Color-coding is a randomized technique that computes simple paths that start and end at two different vertices and no vertex is visited more than once [26]. Given a graph $G$, a set $R$ of a path starting points (e.g. cellular membrane receptors) and a set $T$ of ending points (e.g. transcriptional regulators (TRs)), and a fixed number $l$ representing the path length (number of vertices), the color-coding method randomly assigns to each vertex in the graph a uniformly distributed color (label) from $\{1,2, \ldots, l\}$, and then finds a colorful path that starts at a receptor $\left(v_{1} \in R\right)$, ends at a TR $\left(v_{l} \in T\right)$, and each one of the $l$ vertices composing the path has a distinct color. The constraint of a colorful path (distinct colors of the path vertices) ensures that the reconstructed path is simple. The random designation of colors to the vertices leads to an optimal/sub-optimal solution, if one exists. So, a large number of iterations is required to increase the probability of finding a colorful path. The number of iterations increases exponentially with increasing the probability of success and/or the path length [26]. Enhanced versions of the original color-coding method were proposed to speed up the technique as in [29-31].

The method described in [25] extends the original colorcoding technique [26] by integrating proteins cellular information at reconstructing signaling pathways. To the best of our knowledge, that extended color-coding version [25] (called CC from here on) is the closest in its aim to what we propose in this study. Beside the constraint of a colorful path, $\mathrm{CC}$ allows signaling to advance across the different cellular compartments in a predefined order, i.e. from the cell membrane to the cytosol and then into the nucleus.

LocPL produces $k$ paths: the $k$-shortest paths. In order to compare $L o c P L$ against $C C$, we need $C C$ to produce the same number of paths, where $k=20,000$ in this study. This in turn requires running $\mathrm{CC}$ a number of iterations much larger than $k$ to account for the trials of non-colorful paths. This can take up to days, if not weeks, for a single pathway when the interactions network is very large. The sped up versions of CC mentioned above were tested against relatively smaller networks with hundreds or a few thousands of edges, and many of them may need much modification to integrate the proteins cellular information. So, we augment CC with Yen's algorithm [32] to compute the $k$-shortest paths based on the CC method. We call this the Yen_CC method. Once Yen's algorithm finds a path, it searches for alternative paths that differ from the discovered path in one or more edges. In other words it searches for new partial paths. Hence, in Yen_CC, instead of running a new iteration to find a complete colorful path, the iteration will look for a partial colorful path, leading to reduction in the search space and time. Yen_CC does not handle tied reconstructions, and it reports paths 
with the same reconstruction cost in an arbitrary order in the $k$-paths list. Details about how we implemented the CC method and how we augmented it with Yen's algorithm are provided in the Additional file 1: Section S4.

\section{Interactomes and pathways}

PLNet $_{2}$ Interactome. We built PLNet ${ }_{2}$ from both physical molecular interaction data (BioGrid, DIP, InnateDB, IntAct, MINT, PhosphositePlus) and annotated signaling pathway databases (KEGG, NetPath, and SPIKE) [33-37]. PLNet $_{2}$ contains 17,168 nodes, 40,016 directed regulatory interactions, and 286,250 bidirected physical interactions, totaling 612,516 directed edges. We assigned interaction direction based on evidence of a directed enzymatic reaction (e.g., phosphorylation, dephosphorylation, ubiquitination) from any of the source databases. Each interaction is supported by one or more types of experimental evidence (e.g. yeast two hybrid or co-immunoprecipitation), and/or the name of the pathway database. Edges are weighted using an evidence-based Bayesian approach that assigns higher confidence to an experiment type database if it identifies interacting proteins that participate in the same biological process [9]. Given a set $P$ of positive edges and a set $N$ of negative edges, the method estimates, for each evidence type $t$, the probability that $t$ supports positive interactions. These probabilities are then combined for each interaction supported by (potentially multiple) evidence types to produce a final weight. We chose the GO term "regulation of signal transduction" (GO:0009966) to build a set of positive interactions that are likely related to signaling. Positives are edges whose nodes are both annotated with this term, and negatives are randomly selected edges whose nodes are not co-annotated to the term. We chose $|N|=10 \times|P|$ negative edges. To lessen the influence of very highly-weighted edges, we apply a ceiling of 0.75 to all weights [9].

HIPPIE Interactome. HIPPIE (Human Integrated Protein Protein Interaction rEference) is a repository of 16,707 proteins and 315,484 PPIs [2] (version 2.1, July $18^{\text {th }}, 2017$ [38]). Each interaction has a confidence score calculated as a weighted sum of the number of studies detecting the interaction, the number and quality of experimental techniques used in these studies to measure the interaction, and the number of non-human organisms in which the interaction was reproduced [2]. We ensure that all NetPath interactions are in HIPPIE by using a tool that is provided on the HIPPIE website [38] to integrate new interactions to HIPPIE. We used that tool to score the missed NetPath interactions with the default parameter values used to score the HIPPIE interactions. This lead to adding 792 proteins and 6,379 PPIs to make HIPPIE of 17,499 and 321,863 PPIs in total.
Ground Truth Pathways. We consider a set of four diverse pathways from the NetPath database [35] as our ground truth: $\alpha 6 \beta 4$ Integrin, IL2, EGFR1, and Wnt. Receptors and TRs are automatically detected for each of the eight pathways from lists of 2,124 human receptors and 2,286 human TRs compiled from the literature; see [13] for more details. Additional file 1: Table S1 summarizes the number of interactions, receptors, and TRs per pathway.

\section{Global and path-based assessment}

We assess the performance of LocPL compared to PathLinker $(P L)$ and Yen_CC using two methods that evaluate global and local features of the ranked paths.

Precision-recall (PR) curves. Given a ranked list of paths, we order each interaction by the index of the path in which it first appears. We compute precision and recall for this ranked list using the NetPath interactions as positives and a sampled set of negative interactions that are 50 times the size of the positive set.

Path-based assessment. The PR curves provide a global quantitative assessment across all the $k$ paths in a reconstruction, showing how quickly (in terms of $k$ ) the technique can discover new positive edges. However, this approach considers a positive only once, i.e., the first time it appears in a path. Thus, this global measure fails to characterize each path individually in terms of the number of positives contained in that path. Hence, we introduce a simple way to "locally" assess paths by computing the within-path percentage of true positive edges, denoted as PosFrac. Since we compute this metric value independently for each path, it does not matter if a positive interaction is detected earlier in another path. We compute the PosFrac value over non-overlapping windows of paths. For example, for a window of 100 paths, we compute the average PosFrac over the first 100 paths, then the average PosFrac over the second 100 paths, and so on, providing $k / 100$ values to plot.

Statistical significance. The global assessment is based on two concurrent values: precision and recall. These two quantities are related, so we use their harmonic mean $\left(F_{1}\right.$ score) to get a single value summarizing both values:

$$
F_{1}(i)=2 \times \frac{\text { pre }_{i} \times r e c_{i}}{\text { pre }_{i}+r e c_{i}},
$$

where $\operatorname{pre}_{i}$ and rec $_{i}$ are the $i$-th values of precision and recall, respectively. The $F_{1}$ score values are fed to the Mann-Whitney U (MWU) statistical test for unpaired samples to estimate whether the difference in results between LocPL and PL, and between LocPL and Yen_CC is statistically significant. The inputs to the MWU test for the path-based assessment are the PosFrac values. We 
acknowledge that PosFrac, precision and recall are not purely independent between the two methods, so there is some dependence introduced in the MWU tests.

\section{Results}

\section{Combining interactomes with localization information}

Approximately $95 \%$ of the proteins in $\mathrm{PLNet}_{2}$ have localization information, producing an interactome with about $86 \%$ of the edges (Table 1). Only $65 \%$ of the HIPPIE proteins have localization information, making a much smaller interactome with only about $34 \%$ of the original edges. All pathway receptors and TRs in PLNet $_{2}$ have localization information, and nearly all of them (82 out of 91) in HIPPIE have this information (Additional file 1: Table S1). After filtering PLNet 2 using ComPPI, $62 \%$ of the proteins have a non-zero ExtMem localization score, 78\% have a non-zero Cytosol localization score, and 64\% have a non-zero Nucleus localization score (Additional file 1: Table S2). Most of the proteins have non-zero localization scores for multiple compartments, though $62 \%$ of the proteins with a single non-zero localization score appear in the Nucleus.

Applying PathLinker to the ComPPI-filtered interactome partially mitigates the problem of tied paths, but many ties remain. For example, after running PathLinker on the $\alpha 6 \beta 4$ Integrin pathway with the full $\mathrm{PLNet}_{2}$ interactome, there were 82 groups of paths where each group shared the same reconstruction score (Additional file 1: Table S3). This number was reduced to 58 groups when running PathLinker on the filtered PLNet $_{2}$ interactome. However, ties still dominate the reconstruction scores; thus the need for an approach to breaking these ties and re-prioritizing paths in a biologically relevant way is still imperative.

\section{Assessment of pathway reconstructions}

We applied PathLinker $(P L)$ and LocPL to signaling pathways from the NetPath database to the PLNet $_{2}$ and HIPPIE interactomes as described in the "Interactomes and pathways" subsection. We computed $k=20,000$ paths for each approach, similar to the original publication [13]. Paths that have the same reconstruction score differ substantially in their signaling scores computed by the dynamic program. Figure 3 shows four examples of the signaling score $s_{i}$ distribution for paths with the same reconstruction score $r_{i}$. Signaling scores are

Table 1 Number of proteins and interactions in PLNet 2 and HIPPIE

\begin{tabular}{llllll}
\hline \multirow{2}{*}{ Interactome } & \multicolumn{2}{c}{ Complete Interactome } & & \multicolumn{2}{c}{ Interactome $\cap$ ComPPI } \\
\cline { 2 - 3 } \cline { 6 - 6 } & Nodes & Edges & & Nodes & Edges \\
\hline PLNet 2 & 17,168 & 612,516 & & 16,225 & 527,706 \\
HIPPIE & 17,499 & 321,863 & & 11,430 & 108,391
\end{tabular}

used to re-order paths sharing the same reconstruction score. We also computed 20,000 paths using the Yen_CC approach for the $\mathrm{PLNet}_{2}$ interactome only due to the very long time needed to run Yen_CC. We show results for the PLNet $_{2}$ interactome first and then show those for HIPPIE.

Precision and Recall. We assessed $P L$, LocPL, and $Y e n_{-} C \mathrm{C}$ using the $\mathrm{PLNet}_{2}$ interactome on four signaling pathways: $\alpha 6 \beta 4$ Integrin, EGFR1, IL2, and Wnt. LocPL generally outperforms $P L$ and Yen_CC across all four pathways in terms of precision and recall, where the precision of $L o c P L$ is greater than $P L$ and Yen_CC at nearly all values of recall (Fig. 4 (Left)). Moreover, LocPL usually detects higher proportions of positives than $P L$ and $Y e n_{-} C C$ as reflected in the larger recall values for $L o c P L$ (Fig. 4 (Left)), though the same number of paths were recovered for each method.

For every value of precision and recall, we plotted the harmonic mean $\left(F_{1}\right.$ score) of the two values in Fig. 4 (Right). The $F_{1}$ curve for LocPL is significantly higher than that of $P L$ and Yen_CC for the four pathways (MWU test $p$-value $\leq 0.0001)$.

Assessment of Aggregate Pathways. To assess overall effect of $L o c P L$ on signaling pathway reconstructions, we considered precision and recall aggregated over the four NetPath signaling pathways (Additional file 1: Section S3) for PLNet $_{2}$ (Fig. 5 (left)). LocPL shows better performance over $P L$ and $Y e n_{-} C C$ at nearly all the $k$ values used to compute precision and recall. This improvement is striking at almost all values of recall, with gains in precision that range from $6 \%$ to $32 \%$ at recall of 0.37 and 0.17 , respectively, against PL. When compared to Yen_CC, LocPL achieves gain in precision of about $27 \%$ for recall of 0.1 and on. Superiority of LocPL is significant (MWU test, Fig. 5 (Right)), where the aggregate $F_{1}$ score values are higher everywhere for LocPL.

Path-based Assessment. In addition to the global assessment, we are interested in the quality of subsets of paths. Plotting PosFrac of non-overlapping windows of 100 paths reveals subsets of paths that are enriched for positive interactions in the four pathway reconstructions (Fig. 6). For example, about more than $80 \%$ and $85 \%$ of the paths produced by LocPL for the IL2 pathway reconstruction tend to contain more positive signaling edges than those obtained by $P L$ and Yen_CC, respectively, over all the 20,000 paths. PosFrac is almost consistent for LocPL and, despite some spikes (of different widths) for $P L$ and Yen_CC, PosFrac for LocPL dominates the graph (mean \pm standard deviation values of PosFrac are $0.23 \pm 0.06,0.11 \pm$ 0.12 , and $0.14 \pm 0.07$ for $L o c P L, P L$, and Yen_CC; respectively). In the IL2 pathway reconstruction, this distinction is significant (one-tailed MWU test, Fig. 6). LocPL is also 

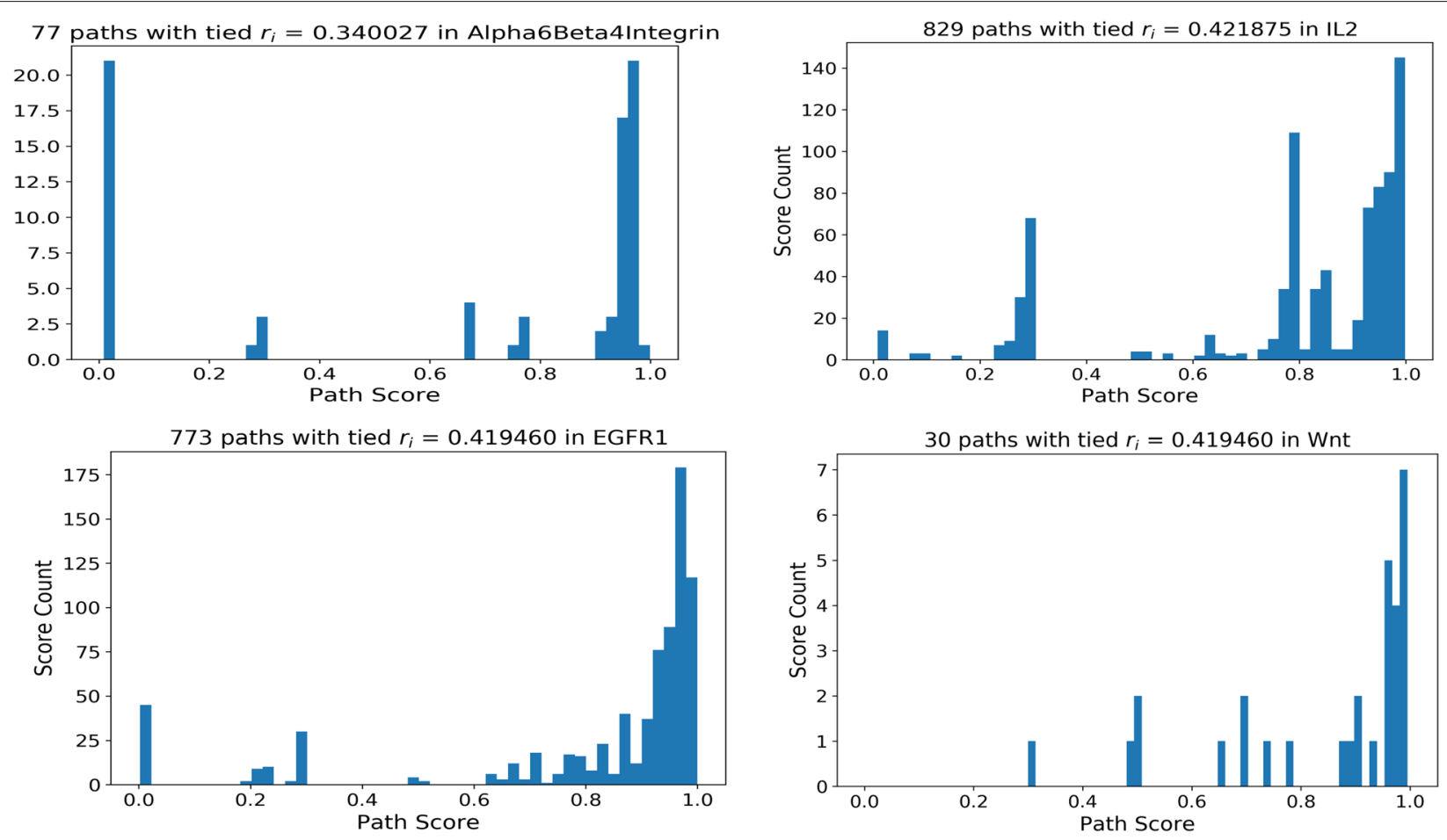

Fig. 3 Histogram of signaling scores $s_{i}$ for paths with tied reconstruction score $r_{i}$. The titles indicate the pathway name, the $r_{i}$ value, and the number of paths tied with this $r_{i}$

significantly better than $P L$ and Yen_CC for the $\alpha 6 \beta 4$ Integrin and EGFR1 pathways. The situation is different for the Wnt pathway, where LocPL is statistically significant when compared against Yen_CC (Fig. 6 (lower right)), but statistically insignificant when tested against PL ( $p$-values of 0.9726, Fig. 6 (lower left)). Note that PosFrac considers all negative interactions for each path, unlike the PR curves in Fig. 4 that subsample the negative set of interactions. Thus, the PosFrac values will be smaller than what one would expect based on the PR curves.

Results on the HIPPIE Interactome. We extended our experiments on the four NetPath signaling pathways ( $\alpha 6 \beta 4$ Integrin, EGFR1, IL2, and Wnt) to the HIPPIE interactome. Figure $7 \mathrm{a}$ (Left) shows, for all the four pathways, that the precision of LocPL is greater than that for $P L$, and that the proportions of positives detected by LocPL is always higher than those of $P L$. This consistently leading performance of LocPL over $P L$ is evidently statistically significant (Fig. 7a (Right)). Again, the aggregate precision of LocPL has gains of up to $40 \%$ over that of $P L$, and the recall proportion is more than the double for LocPL (Fig. 7c). The reconstructed paths of $L o c P L$ are steadily and significantly more enriched with positive interactions than the paths of $P L$ (Fig. 7b).

\section{Comparison of pathway reconstructions}

LocPL provides a compartment-aware ranking of paths connecting receptors to TRs. In addition to the global and local assessments provided above, we examined the 100 top-ranking paths of $P L, L o c P L$, and Yen_CC pathway reconstructions using $\mathrm{PLNet}_{2}$ for the $\alpha 6 \beta 4$ Integrin, IL-2, EGFR1, and Wnt pathways. We first counted the number of paths with at least one positive interaction and the number of paths whose all interactions are positives within the first 10 and 100 paths. In most of the cases, LocPL identifies more positive-enriched paths than $P L$ and $Y e n_{-} C C$ (Table 2). Note that the number of positives in the earliest paths for the Wnt pathway is larger for $P L$ over LocPL, which agrees with the PosFrac values shown in Fig. 6 (lower left).

We then wished to better understand how the constraints imposed by the dynamic program affected the pathway reconstructions. We compared the subgraph comprised of the first 100 paths before applying the dynamic program that reorders ties based on signaling score, to the subgraph comprised of the first 100 paths after applying the dynamic program. While the number of nodes and edges were about the same between the two subgraphs, we found that EGFR1, IL2, and Wnt only had about half the number of nodes in common and about a third the number of edges in common (Additional file 1: Figure S2). The number of common nodes and edges for 

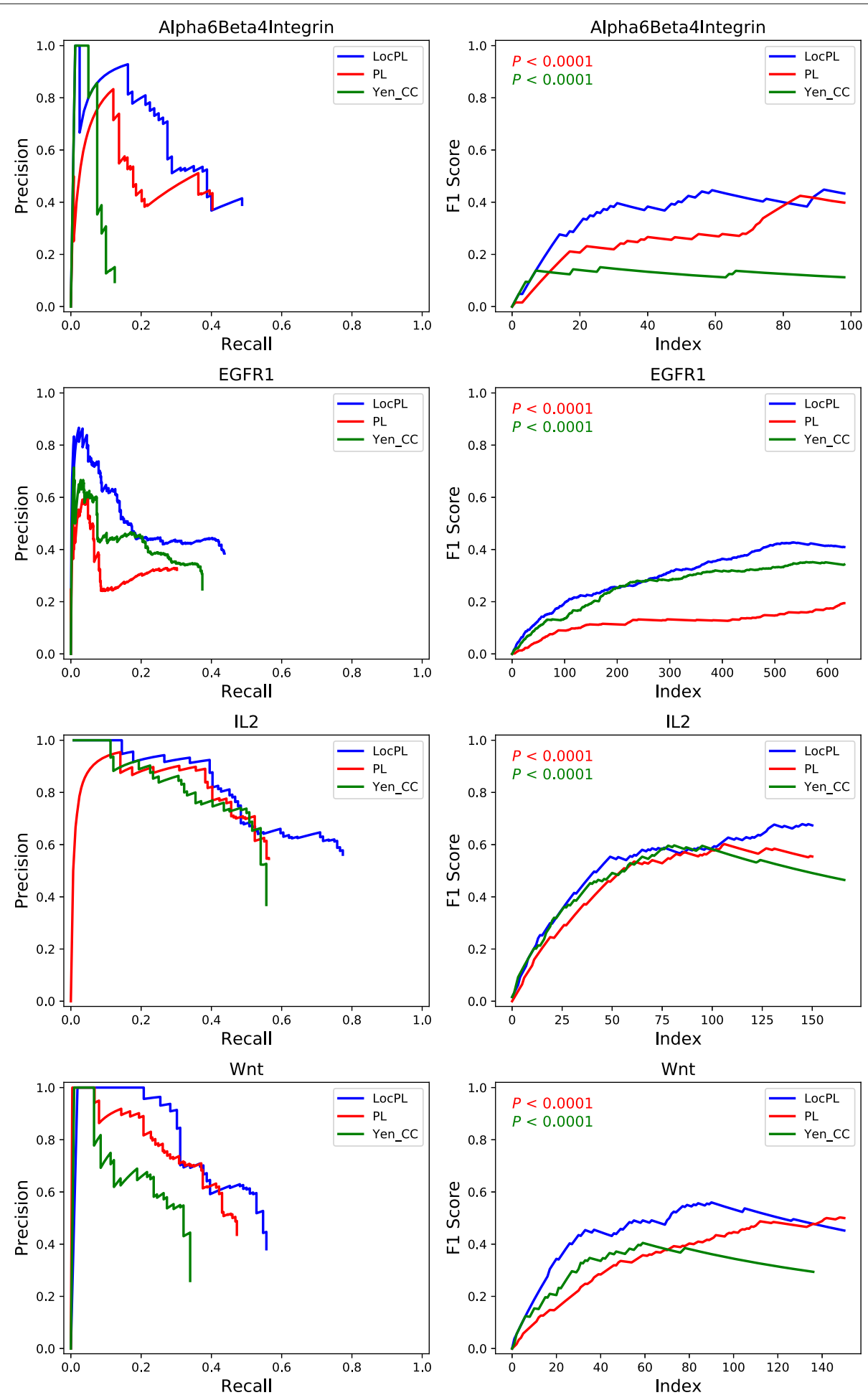

Fig. 4 PLNet 2 : (Left) Precision and recall curves of pathway reconstructions from PathLinker (PL), LocPL, and Yen_CC on four NetPath signaling pathways. (Right) $F_{1}$ scores for the individual NetPath pathways. These values are fed to the MWU test to check for difference significance. The $p$-value, $P$, is for the MWU test (alternative: $L O C P L>P L$ or $L O C P L>Y e n \_C C$ ). The color of the $p$-value text indicates which method is tested against $L O C P L$, e.g. the red text tests that the $F_{1}$ score of $L O C P L$ is greater than that of $P L$ 


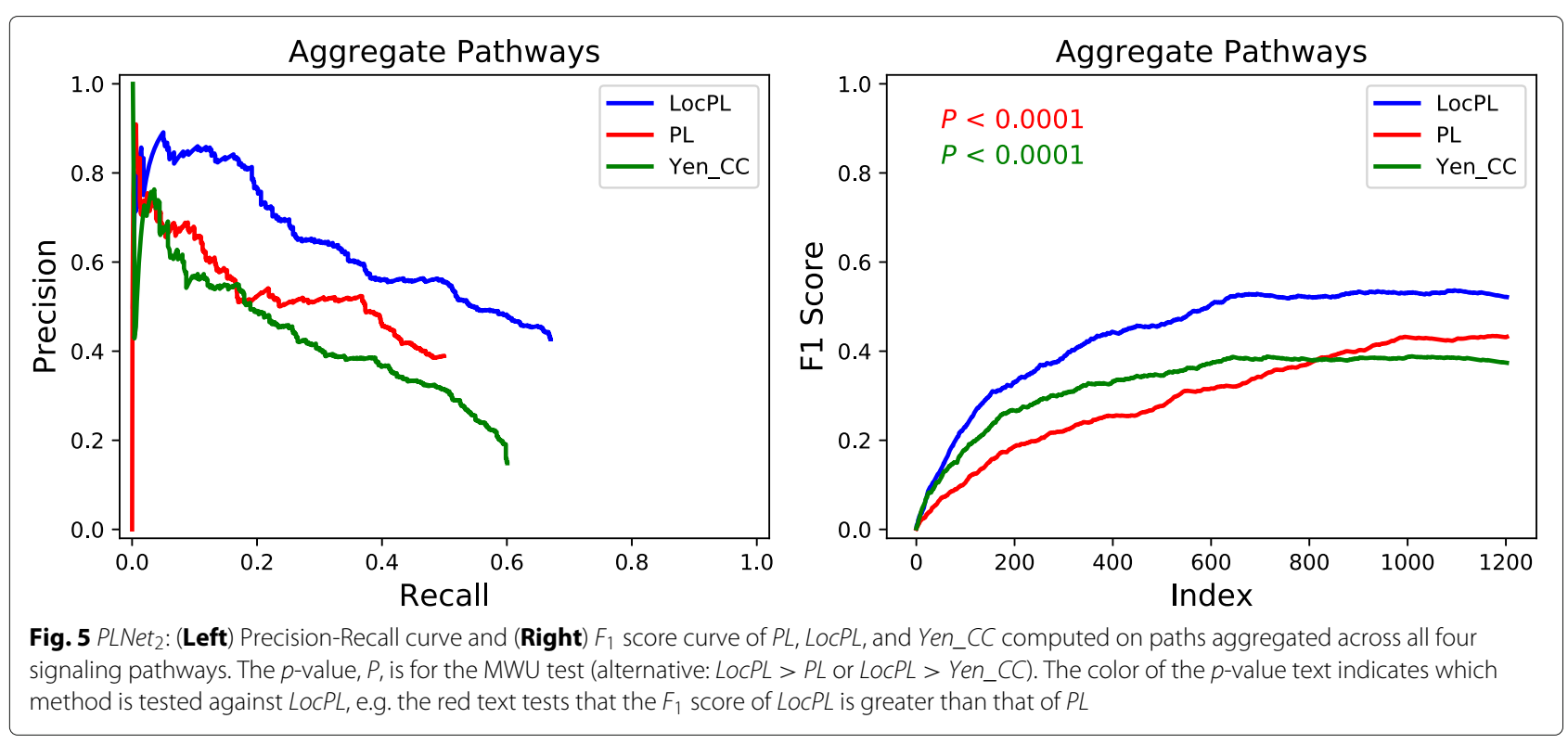

the two subgraphs of $\alpha 6 \beta 4$ Integrin are about, at least, double the number of the unique nodes and edges to either subgraph.

We also visualized networks for each pathway reconstruction before and after applying the dynamic program (Fig. 8). The nodes are colored according to red, green, and blue channels depending on the ComPPI localization scores for membrane, cytosol, and nucleus respectively; a protein that appears in all compartments will be white. The signaling flow constraints from the dynamic program on LocPL paths imply two features about these networks: first, the node colors should change from red (membrane) to green (cytosol) to blue (nucleus), and second, no paths of length one are allowed. Both of these features are visible in the comparison of the IL2 pathway reconstructions (Fig. 8a). For example, the edge from IL2 Receptor A (IL2RA) to transcription factor STAT5B is removed after the dynamic program, removing the IL2RA receptor from the first 100 paths.

The color differences between the two IL2 networks are also notable. Before the dynamic program, the IL2 reconstruction contains main proteins that are predicted to be at the membrane, including the IL7 receptor (IL7R), Insulin Like Growth Factor 1 Receptor (IGF1R), Leptin Receptor (LEPR), KIT Proto-Oncogene Receptor Tyrosine Kinase (KIT), and Erythropoietin Receptor (EPOR). Further, the Interleukin 6 Signal Transducer (IL6ST) is also reported to be at the membrane, yet is downstream of Suppressor Of Cytokine Signaling 3 (SOCS3) in the network (Fig. 8a (Left)). IL2 signaling activates the Jak/STAT pathway, and many paths containing Janus kinase family members (JAK1, JAK2, JAK3) also include SOCS3 upstream of these proteins. After the paths are reordered according to the dynamic program, the JAK proteins are directly dosntream of the receptors (Fig. 8a (Right)). While some receptors remain after reordering, they either directly interact with the IL2 receptors (e.g. IL7R), or they lie downstream of a protein that is consistent in terms of the signaling constraints. For example, the the SYK-FGR is allowable because SYK has a large ComPPI score for all compartments. The other pathways exhibit dramatic differences in topology compared to the IL2 reconstructions, including the large number of receptors in the Wnt reconstructions, the large number of TFs in the EGFR1 reconstructions, and the large number of intermediate nodes in the Alpha6 $\beta 4$ Integrin reconstruction (Fig. $8 \mathrm{~b}$ in this text and Additional file 1: Figures S3, S4 and S5).

\section{Discussion}

We present $L o c P L$, an automatic signaling reconstruction algorithm that incorporates information about protein localization within the cell. Previous reconstructions contained many tied paths. LocPL overcomes this obstacle with a computational framework that favors paths that follow specific assumptions of signaling flow. This framework includes filtering interactions based on their predicted interaction score and applying a dynamic program to each path that finds the most likely series of cellular compartments that are consistent with the model of signaling flow.

Using a new interactome, PLNet $_{2}$, we have shown that LocPL pathway reconstructions for four pathways are more enriched with positive interactions than paths computed by $P L$ and by a peer method, Yen_CC, based on the color coding technique. Precision of LocPL dominates the precision of $P L$ and Yen_CC at nearly every value of recall (Fig. 4 (Left)), and the resulting $F_{1}$ scores are 

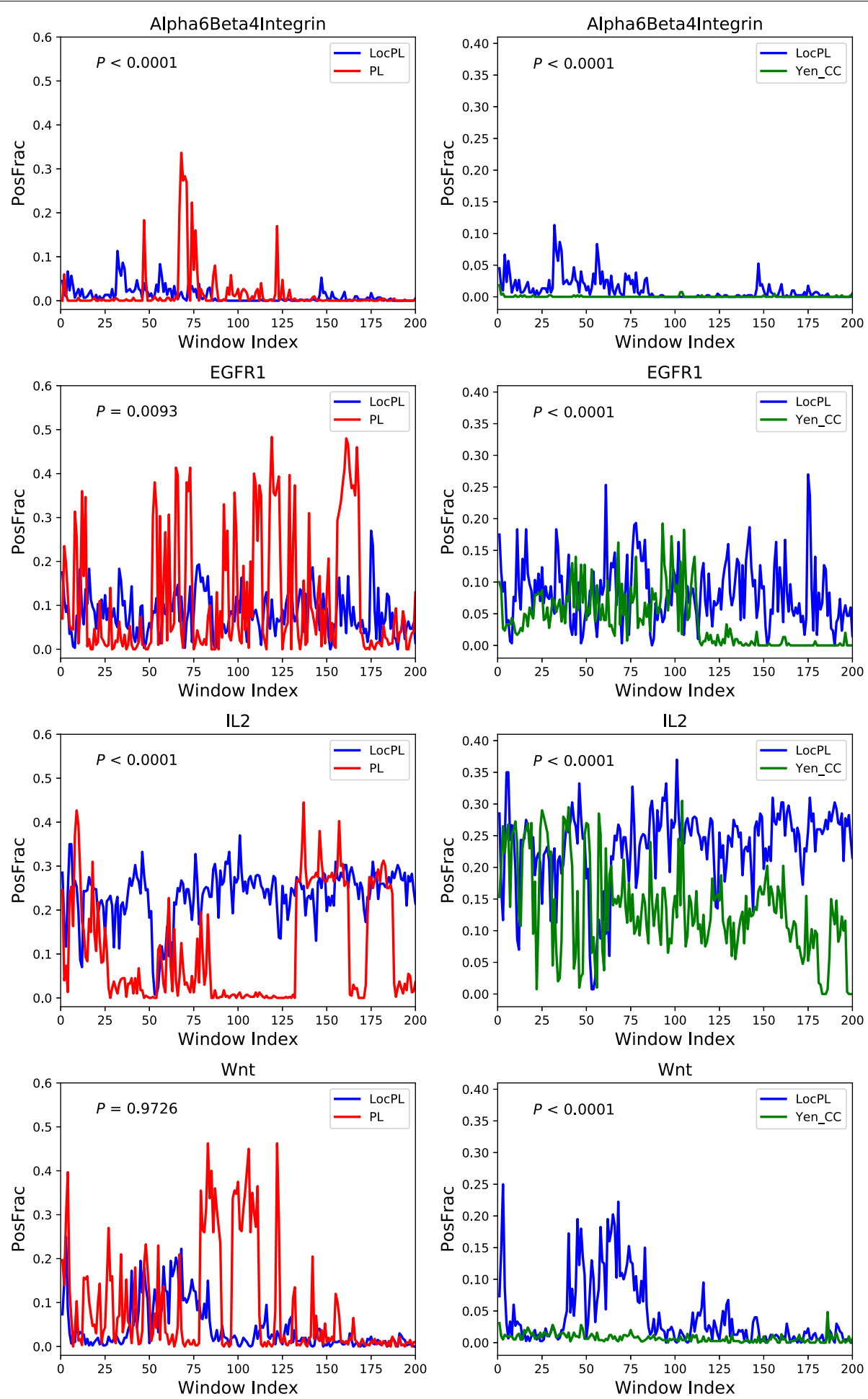

Fig. 6 PLNet2: Path-based performance of four NetPath signaling pathways for (Left) LocPL vs. PL and (Right) LocPL vs. Yen_CC. PosFrac is the percentage of positives averaged across non-overlapping windows of 100 paths. The $p$-value, $P$, is for the MWU test (alternative: LocPL > PL or LocPL $>$ Yen_(C) 

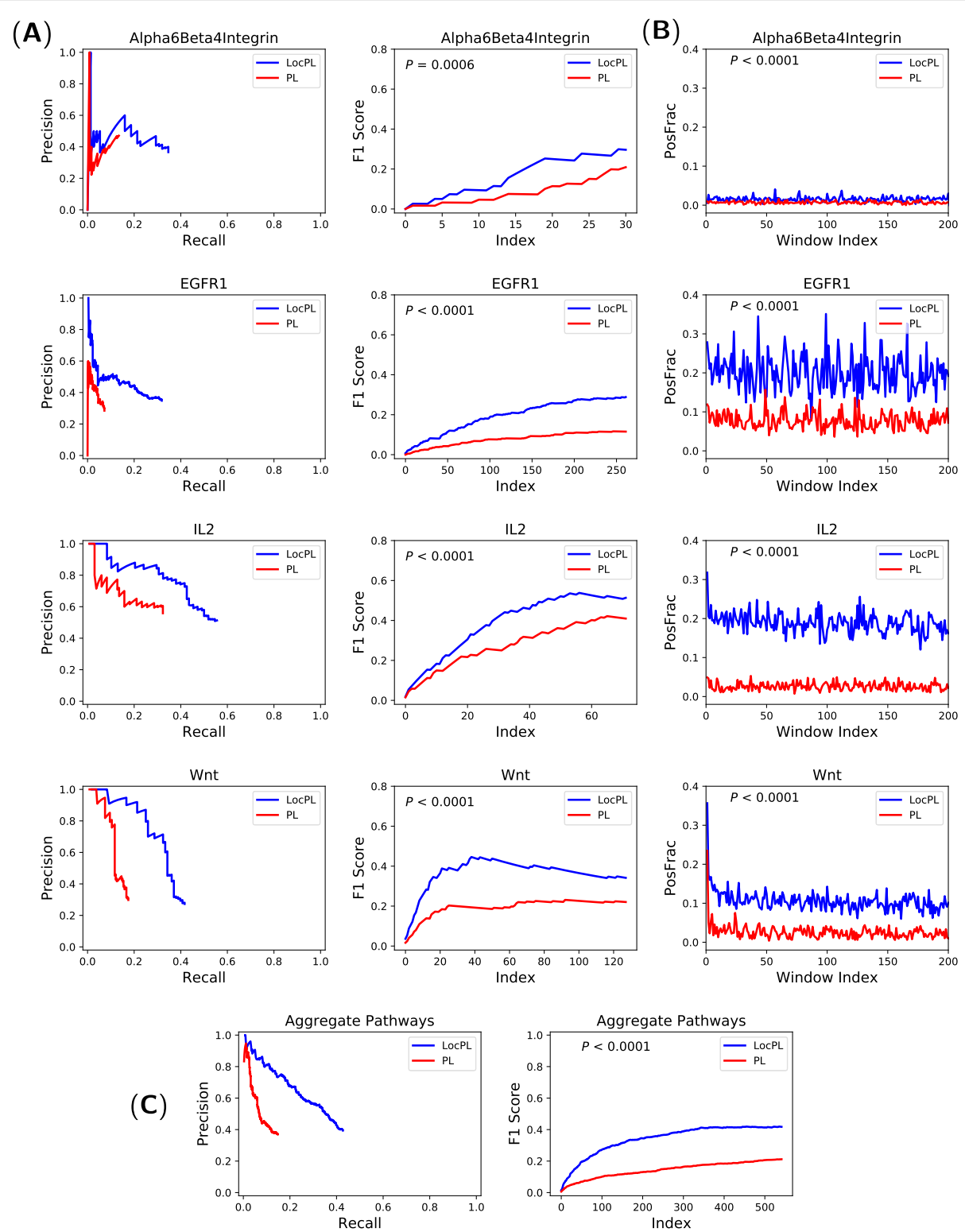

Fig. 7 HIPPIE: (a: Left) Precision and recall curves of pathway reconstructions from PathLinker $(P L)$ and LocPL on four NetPath signaling pathways. (a: Right) $F_{1}$ scores for the individual NetPath pathways. b Path-based performance of the individual pathways. PosFrac is the percentage of positives averaged across non-overlapping windows of 100 paths. (c: Left) Aggregate PR curve, and (c: Right) $F_{1}$ score curve over the four signaling pathways. The $p$-value, $P$, is for the MWU test (alternative: $L O C P L>P L$ )

significantly better for LocPL (Fig. 4 (Right)). LocPL dramatically improves precision at all values of recall across four signaling pathways, and this difference is significant by the MWU test (Fig. 5).

In addition to the precision and recall assessment used previously by PathLinker [13], we proposed a measure, PosFrac, to assess individual paths in terms of proportion of positive signaling interactions. PR curves demonstrate how quickly positive interactions are recovered in a reconstruction, but do not consider the fact that many paths may contain the same positive. PosFrac is a path-based measure that considers the proportion of positives within a set of paths, demonstrating that some sets of paths are enriched for positive interactions that may have appeared in a higher-ranked path. LocPL paths are consistently enriched with positive interactions more than the paths reconstructed by $\mathrm{Yen}_{-} \mathrm{CC}$ for all the four signaling pathways, and more than the paths of $P L$ for two of the pathways (Fig. 6). This measure offers complementary insights to the pathway reconstructions beside the PR curves. For example, paths within windows 50 to 65 for the IL2 pathway (Fig. 6) have very small PosFrac values among 
Table $2 \mathrm{PLNet}_{2}$ : The number of paths with at least one positive interaction (partial) and with all interactions are positives (complete) among the first 10 and 100 reconstructed paths

\begin{tabular}{|c|c|c|c|c|}
\hline \multirow{2}{*}{ Pathway/Method } & \multicolumn{2}{|c|}{ First 10 Paths } & \multicolumn{2}{|c|}{ First 100 Paths } \\
\hline & Partial & Complete & Partial & Complete \\
\hline \multicolumn{5}{|l|}{$\alpha 6 \beta 4$ Integrin } \\
\hline$P L$ & 0 & 0 & 0 & 0 \\
\hline LOCPL & 1 & 0 & 9 & 0 \\
\hline Yen_CC & 1 & 0 & 4 & 0 \\
\hline \multicolumn{5}{|l|}{ EGFR1 } \\
\hline$P L$ & 0 & 0 & 11 & 3 \\
\hline LOCPL & 1 & 0 & 29 & 6 \\
\hline Yen_CC & 2 & 0 & 20 & 0 \\
\hline \multicolumn{5}{|l|}{ IL2 } \\
\hline$P L$ & 0 & 0 & 39 & 8 \\
\hline LOCPL & 5 & 0 & 44 & 6 \\
\hline Yen_CC & 3 & 1 & 30 & 5 \\
\hline \multicolumn{5}{|l|}{ Wnt } \\
\hline$P L$ & 3 & 0 & 32 & 6 \\
\hline LOCPL & 6 & 4 & 13 & 4 \\
\hline Yen_CC & 3 & 2 & 5 & 2 \\
\hline
\end{tabular}

all the 20,000 paths. These paths contain interactions that are not labeled as positives but are "close" to the pathway in some sense, suggesting candidate interactions that may point to non-canonical branches of signaling.

Though both LocPL and the color coding method (CC, [25]) use protein localization information, but the way this information is employed differs substantially. $C C$ uses a binarized version of the localization information; what cellular compartments a protein can be found within. This leads to tied reconstructions due to the deprivation from having other measures, beside the reconstruction cost, to re-prioritize ties. In contrast, LocPL uses a probabilistic form of the localization information; the likelihood of a protein to be found in one cellular compartment. This furnishes $L o c P L$ with a second measure, the signaling score, to untangle ties and re-order reconstructions.

LocPL ensures that the constituting interactions, from a receptor to a TR, are spatially-coherent within the different cellular compartments. This feature increases the number of paths that contain positives early in the pathway reconstruction, which supports our hypothesis that LocPL locally promotes paths with higher proportions of positives up in the $k$-shortest paths list (Table 2).

$L O c P L$ is not restricted to our proposed interactome, PLNet $_{2}$. We applied LocPL to the HIPPIE interactome [2]. We compared $L o c P L$ to only $P L$ due to the very long time demand of the Yen_CC method. LocPL's performance was statistically significantly better than $P L$ as depicted in the PR and the $F_{1}$ score curves (Fig. 7a) and in the PosFrac curves (Fig. 7b) for the individual NetPath signaling pathways. Moreover, this trend is consistent across the four signaling pathways as well (Fig. 7c).

In this work, we chose to impose an ordering on a subset of the available compartments from ComPPI (ExtMem, Cytosol, and Nucleus). There are many ways to impose a compartmental ordering of signaling flow to capture other features of signaling, including mitochondria-dependent signaling, nuclear receptor signaling and extracellular signaling. LocPL is generalizable to different signaling models, as long as the user specifies compartment relationships in a memoryless manner (the signaling score at the next node depends only on the localization score of the next node and the signaling score at the current node; ignoring signaling score history at previous nodes). To illustrate this point, we developed a model of signaling that also includes the mitochondria compartment. We did not notice any changes in the results when we included the mitochondria into our signaling model, most likely due to the relatively few number of proteins in $\mathrm{PLNet}_{2}$ that had non-zero Mitochondria localization scores (Additional file 1: Table S2). Details about how this modified signaling model and the dynamic program can be found in Additional file 1: Section S2.

Visual inspection of the subgraphs containing the first 100 paths in the pathway reconstructions before and after applying the dynamic program reveal that reordering tied paths changes the first 100 paths dramatically, even though the number of nodes and edges remain similar (Additional file 1: Figure S2). In particular, the dynamic program removes membrane-bound receptors that appear downstream of cytosolic proteins, which can be seen by visual inspection (Fig. 8). These and other features can be explored in such network reconstructions.

\section{Conclusion}

In this study, we presented LocPL, which is a powerful tool for automatic reconstruction of signaling pathways from protein-protein interactions that leverages the proteins cellular localization information. LocPL showed profound and significant better reconstructions over those by peer methods in terms of the total number of the true protein interactions across the whole pathway reconstructions and the number of positive interactions per individual paths with a reconstruction. The framework that we have developed may be extended to other graph-theoretic approaches that return subnetworks of directed structure with an associated reconstruction score, such as trees $[10,11,15]$. Our approach encourages the enumeration of many tied results, since incorporating protein compartment information will help break these ties with biologically relevant information. In addition, we anticipate to develop the technique to compare paths in different contexts, such as tissue-specific or disease-specific signaling. 


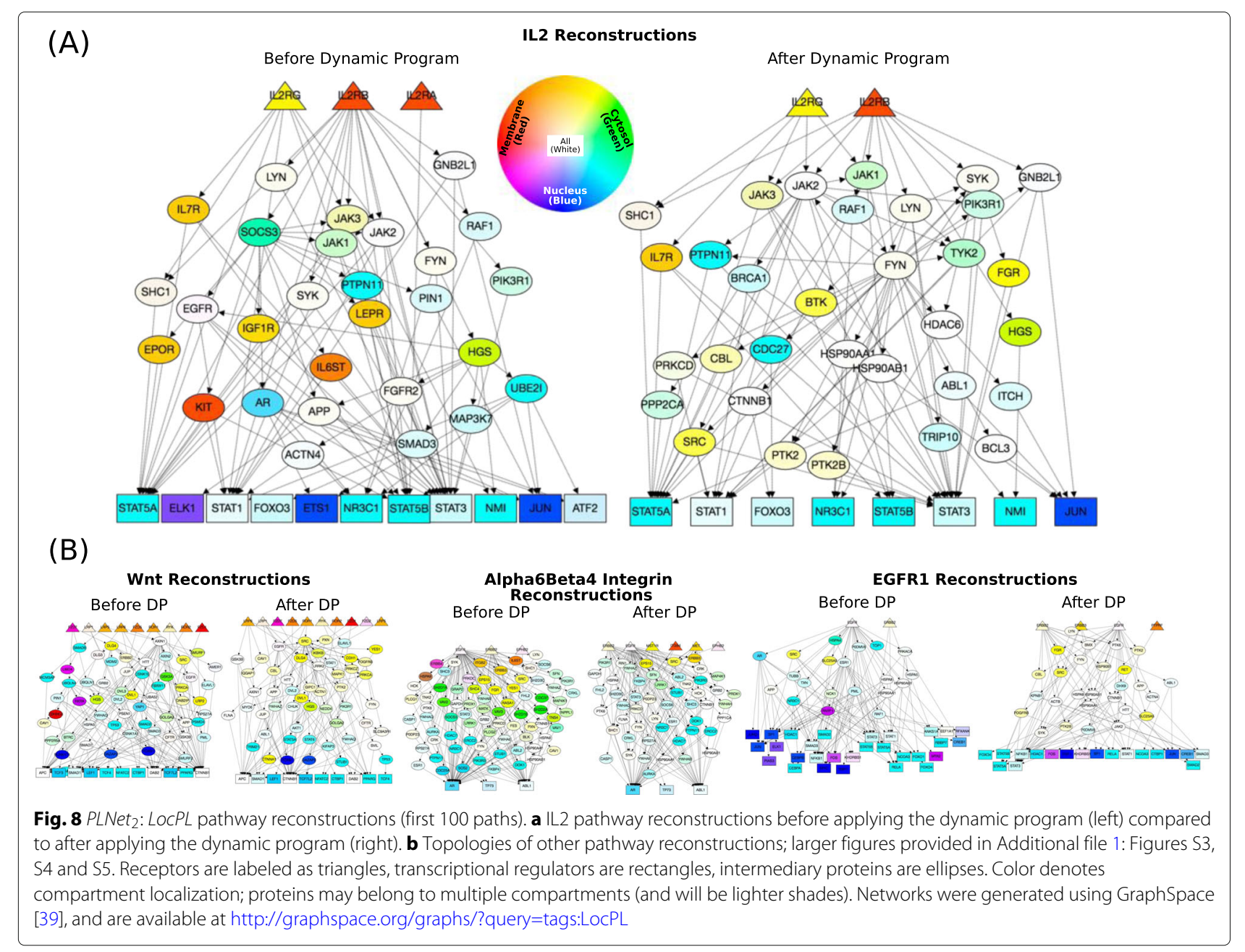

\section{Supplementary information}

Supplementary information accompanies this paper at

https://doi.org/10.1186/s12859-019-3077-x.

Additional file 1: This file includes the additional material referred to through the main manuscript.

\section{Acknowledgements}

We thank T.M. Murali for useful discussions about PathLinker and other pathway reconstruction methods.

\section{About this supplement}

This article has been published as part of BMC Bioinformatics Volume 20 Supplement 16, 2019: Selected articles from the IEEE BIBM International Conference on Bioinformatics \& Biomedicine (BIBM) 2018: bioinformatics and systems biology. The full contents of the supplement are available online at https:// bmcbioinformatics.biomedcentral.com/articles/supplements/volume-20supplement- 16 .

\section{Authors' contributions}

AR supervised the project. AR and IY conceived of the project and developed the LOCPL algorithm. JL generated and weighted the new PLNet2 network. IY implemented the methods and conducted all experiments. All authors contributed to the writing and editing of the manuscript. All authors read and approved the final manuscript.

\section{Funding}

This work was supported by the National Science Foundation (NSF) grant (ABI-1750981, awarded to AR) and a College Research Program for Natural Sciences grant by the M. J. Murdock Charitable Trust (awarded to AR). JL was partially supported by the National Institute of General Medical Sciences (R01-GM095955-01) and by the Office of the Director of National Intelligence (ODNI), Intelligence Advanced Research Projects Activity (IARPA), via the Army Research Office (ARO) under cooperative Agreement Number W911NF-17-2-0105. The views and conclusions contained herein are those of the authors and should not be interpreted as necessarily representing the official policies or endorsements, either expressed or implied, of the ODNI, IARPA, ARO, or the U.S. Government. The U.S. Government is authorized to reproduce and distribute reprints for Governmental purposes notwithstanding any copyright annotation thereon. Publication costs were funded by the NSF grant (ABI-1750981).

\section{Availability of data and materials}

All code and input files, including the newly-released interactome $\mathrm{PLNet}_{2}$, are available at https://github.com/annaritz/localized-pathlinker.

\section{Ethics approval and consent to participate}

Not applicable.

\section{Consent for publication}

Not applicable. 


\section{Competing interests}

The authors declare that they have no competing interests.

\section{Author details \\ ${ }^{1}$ Biomedical Engineering Department, Cairo University, Giza, 12613, Egypt. ${ }^{2}$ Biology Department, Reed College, Portland OR 97202, USA. ${ }^{3}$ Genetics, Bioinformatics, and Computational Biology, Virginia Tech, Blacksburg VA 24061, USA.}

\section{Published: 2 December 2019}

\section{References}

1. Schaefer M, Fontaine J, Vinayagam A, Porras P, Wanker E, Andrade-Navarro M. HIPPIE: Integrating protein interaction networks with experiment based quality scores. PLoS ONE. 2012;7(2):e31826.

2. Alanis-Lobato G, Andrade-Navarro M, Schaefer M. HIPPIE v2.0: enhancing meaningfulness and reliability of protein-protein interaction networks. Nucleic Acids Res. 2017;45:D408-14.

3. Das J, Yu H. HINT: High-quality protein interactomes and their applications in understanding human disease. BMC Syst Biol. 2012;6:92.

4. Li T, Wernersson R, Hansen RB, Horn H, Mercer J, et al. A scored human protein-protein interaction network to catalyze genomic interpretation. Nat Methods. 2017;14(1):61-4

5. Chatr-aryamontri A, Tyers M, Theesfeld C, Chang C, Rust J, Dolinski K, et al. The BioGRID interaction database: 2017 update. Nucleic Acids Res. 2017;45(D1): D369-79. Available from https://dx.doi.org/10.1093/nar/gkw1102.

6. Stutz A, Melidoni AN, Raghunath A, Lagreid A, Roechert B, Meldal B, et al. The MIntAct project-IntAct as a common curation platform for 11 molecular interaction databases. Nucleic Acids Res. 2014;42(D1):D358-63. Available from: https://dx.doi.org/10.1093/nar/gkt1115.

7. Roth A, SzklarczykD, Simonovic M, Wyder S, von Mering C, Morris JH, et al. The STRING database in 2017: quality-controlled protein-protein association networks, made broadly accessible. Nucleic Acids Res. 2017;45(D1): D362-8. Available from: https://dx.doi.org/10.1093/nar/gkw937.

8. Huang S, Fraenkel E. Integrating Proteomic, Transcriptional, and Interactome Data Reveals Hidden Components of Signaling and Regulatory Networks. Sci Signal. 2009;2(81:ra40):. Available from: http:// stke.sciencemag.org/content/2/81/ra40.

9. Yeger-Lotem E, Riva L, Su L, Gitler A, Cashikar A, et al. Bridging high-throughput genetic and transcriptional data reveals cellular responses to alpha-synuclein toxicity. Nat Genet. 2009;41(3):316-23.

10. Bailly-Bechet M, Borgs C, Braunstein A, Chayes J, Dagkessamanskaia A et al. Finding undetected protein associations in cell signaling by belief propagation. Proc Natl Acad Sci. 2011;108(2):882-7. Available from: http://www.pnas.org/content/108/2/882.

11. Yosef N, Ungar L, Zalckvar E, Kimchi A, Kupiec M, et al. Toward accurate reconstruction of functional protein networks. Mol Syst Biol. 2009;5(1): Available from: http://msb.embopress.org/content/5/1/248.

12. Supper J, Spangenberg L, Planatscher H, Drager A, Schroder A, Zell A. BowTieBuilder: modeling signal transduction pathways. BMC Syst Biol. 2009;3:67.

13. Ritz A, Poirel C, Tegge A, Sharp N, Simmons K, Powell A, et al. Pathways on demand: automated reconstruction of human signaling networks. npj Syst Biol Appl. 2016;2:16002.

14. Moon JH, Lim S, Jo K, Lee S, Seo S, Kim S. PINTnet: construction of condition-specific pathway interaction network by computing shortest paths on weighted PPI. BMC Syst Biol. 2017;1 (Suppl 2):15. Available from: http://www.ncbi.nlm.nih.gov/pmc/articles/PMC5374644/.

15. Tuncbag N, Braunstein A, Pagnani A, Huang S, Chayes J, et al. Simultaneous reconstruction of multiple signaling pathways via the prize-collecting steiner forest problem. J Comput Biol. 2013;20(2):124-36.

16. Sun $Y, M a C$, Halgamuge $S$. The node-weighted Steiner tree approach to identify elements of cancer-related signaling pathways. BMC Bioinformatics. 2017;18(Suppl 16):551. Available from: http://www.ncbi. nlm.nih.gov/pmc/articles/PMC5751691/.

17. Nassiri I, Masoudi-Nejad A, Jalili M, Moeini A. Discovering dominant pathways and signal-response relationships in signaling networks through nonparametric approaches. Genomics. 2013;102(4):195-201. Available from: http://www.sciencedirect.com/science/article/pii/ S0888754313001493.
18. Kittas A, Delobelle A, Schmitt S, Breuhahn K, Guziolowski C, Grabe N. Directed random walks and constraint programming reveal active pathways in hepatocyte growth factor signaling. FEBS J. 2016;283(2):350-60. Available from: https://febs.onlinelibrary.wiley.com/doi/abs/10.1111/febs.13580.

19. Sun J, Zhao M, Jia P, Wang $L$, Wu Y, et al. Deciphering Signaling Pathway Networks to Understand the Molecular Mechanisms of Metformin Action. PLoS Comput Biol. 2015;1 1(6):1-35. Available from: https://doi.org/10.1371/journal.pcbi.1004202.

20. Paull E, Carlin D, Niepel M, Sorger P, Haussler D, Stuart J. Discovering causal pathways linking genomic events to transcriptional states using Tied Diffusion Through Interacting Events (TieDIE). Bioinformatics. 2013;29(21):2757-64.

21. Nusse R, Clevers H. Wnt/ $\beta$-Catenin Signaling, Disease, and Emerging Therapeutic Modalities. Cell. 2017;169(6):985-99. Available from: http:// dx.doi.org/10.1016/j.cell.2017.05.016.

22. Arenas-Ramirez N, Woytschak J, Boyman O. Interleukin-2: Biology, Design and Application. Trends Immunol. 2015;36(12):763-77. Available from: http://www.sciencedirect.com/science/article/pii/S1471490615002483.

23. Berg K, Lange T, Mittelberger F, Schumacher U, Hahn U. Selection and Characterization of an $\alpha 6 \beta 4$ Integrin blocking DNA Aptamer. Mol Ther Nucleic Acids. 2016;5:e294. Available from: http://www.sciencedirect. com/science/article/pii/S2162253117300239.

24. Fromm JA, Johnson SAS, Johnson DL. Epidermal Growth Factor Receptor 1 (EGFR1) and Its Variant EGFRvIll Regulate TATA-Binding Protein Expression through Distinct Pathways. Mol Cell Biol. 2008;28:6483-95. Available from: http://www.ncbi.nlm.nih.gov/pmc/articles/PMC2577416/.

25. Scott J, Ideker T, Karp RM, Sharan R. Efficient Algorithms for Detecting Signaling Pathways in Protein Interaction Networks. J Comput Biol. 2006;13(2): 133-44. Available from: https://doi.org/10.1089/cmb.2006.13.133.

26. Alon N, Yuster R, Zwick U. Color-coding. J ACM. 1995;42(4):844-56. Available from: http://doi.acm.org/10.1145/210332.210337.

27. Veres D, Gyurko M, Thaler B, Szalay K, Fazekas D, et al. ComPPI: a cellular compartment-specific database for protein-protein interaction network analysis. Nucleic Acids Res. 2015;43(D1):D485-93.

28. The Compartmentalized Protein-Protein Interaction Database. 2018. Available from: http://comppi.linkgroup.hu/. Accessed 11 Dec 2018.

29. Hüffner F, Wernicke S, Zichner T. Algorithm Engineering for Color-Coding with Applications to Signaling Pathway Detection. Algorithmica. 2008;52(2):114-32. Available from: https://doi.org/10.1007/ s00453-007-9008-7.

30. Yeh CY, Yeh HY, Arias CR, Soo VW. Pathway Detection from Protein Interaction Networks and Gene Expression Data Using Color-Coding Methods and A* Search Algorithms. Sci World J. 2012. https://doi.org/10. 1100/2012/315797

31. Slota G, Madduri K. Parallel color-coding. Parallel Comput. 2015;47:51-69.

32. Yen JY. Finding the K Shortest Loopless Paths in a Network. Manag Sci. 1971;17(11):712-6. Available from: http://www.jstor.org/stable/2629312.

33. Aranda B, Blankenburg H, Kerrien S, Brinkman F, Ceol A, et al. PSICQUIC and PSISCORE: accessing and scoring molecular interactions. Nat Methods. 2011;8(7):528-9.

34. Hornbeck P, Kornhauser J, Tkachev S, Zhang B, Skrzypek E, et al. PhosphoSitePlus: a comprehensive resource for investigating the structure and function of experimentally determined post-translational modifications in man and mouse. Nucleic Acids Res. 2012;40(Database issue):D261-70.

35. Kandasamy K, Mohan S, Raju R, Keerthikumar S, Kumar G, et al. NetPath: a public resource of curated signal transduction pathways. Genome Biol. 2010;11(1):R3.

36. Kanehisa M, Furumichi M, Tanabe M, Sato Y, Morishima K. KEGG: new perspectives on genomes, pathways, diseases and drugs. Nucleic Acids Res. 2017:45(D1):D353-61.

37. PazA, Brownstein Z, Ber Y, BialikS, David E, et al. SPIKE: a database of highly curated human signaling pathways. Nucleic Acids Res. 2011;39(suppl 1):D793-9.

38. The Human Integrated Protein-Protein Interaction rEference. 2018. Available from: http://cbdm-01.zdv.uni-mainz.de/ mschaefer/hippie/ index.php. Accessed 13 Aug 2017.

39. Bharadwaj A, Singh D, Ritz A, Tegge A, Poirel C, et al. GraphSpace: stimulating interdisciplinary collaborations in network biology. Bioinformatics. 2017;33(19):3134-6.

\section{Publisher's Note}

Springer Nature remains neutral with regard to jurisdictional claims in published maps and institutional affiliations. 